\title{
TRACKING CONTROL FOR UNCERTAIN TAKAGI SUGENO FUZZY SYSTEMS WITH EXTERNAL DISTURBANCES
}

\author{
Badr Mansouri, Noureddine Manamanni, Abdelaziz Hamzaoui and Janan Zaytoon \\ CReSTIC Université de Reims Champagne-Ardenne \\ UFR Sciences Exactes et Naturelles \\ Moulin de la Housse BP 1039 (Bâtiment 12) 51687 REIMS cedex 2 - FRANCE \\ \{badr.mansouri,noureddine.manamanni, abdelaziz.hamzaoui,janan.zaytoon\}@univ-reims.fr
}

\begin{abstract}
In this paper a fuzzy tracking control is proposed for non linear dynamic system with bounded external disturbances and admissible uncertainties described as TakagiSugeno (T-S) fuzzy model. Using a tracking reference model, a stability condition scheme based on an augmented system with a guaranteed $\mathrm{H} \infty$ performance is proposed to obtain tracking performances. The main result of this paper is to provide, for the overall system in the presence of uncertainties, a sufficient stability condition for the robust fuzzy $\mathrm{H} \infty$ controller expressed in terms of linear matrix inequalities (LMIs). An example of robust fuzzy $\mathrm{H} \infty$ controller for uncertain nonlinear system is given for illustration. Copyright @ 2005 IFAC
\end{abstract}

Keywords: Fuzzy control, linear matrix inequality (LMI), quadratic stability, tracking, relaxed condition, $\mathrm{H} \infty$ robust control, uncertain systems.

\section{INTRODUCTION}

An essential problem in control theory is that of controlling a system in order to have its output asymptotically tracking reference signals. For nonlinear dynamic systems, it is not obvious to solve tracking problems. Hence, stabilisation problems are often treated first. In general, these problems for such kind of systems use special control strategies like sliding mode control, fuzzy adaptive control and so on. Several works have recently focused on the stabilization of nonlinear dynamic systems using Takagi-Sugeno (T-S) fuzzy models (Kim, et al., 1995; Narendra and Annaswamy, 1989; Sastry and Bodson, 1989; Takagi and Sugeno, 1985; Wang, et al., 1996).

It is well known that T-S models can provide an efficient representation of complex nonlinear systems in terms of fuzzy sets and fuzzy reasoning (Takagi and Sugeno, 1985). These kinds of models have been proved to be a very good representation of a certain class of non linear systems. The appeal of the T-S model is that the stability and performance characteristics of the system can be analysed using a Lyapunov approach. It has been demonstrated (Kim, et al., 1995; Wang, et al., 1996) that sufficient conditions for the stability and performances of a system are stated in terms of a linear matrix inequalities (LMIs) set. The control design is carried out based on the fuzzy model via the so-called parallel-distributed compensation scheme (PDC) (Wang, et al., 1995; Wang, et al., 1996). On the one hand, most of the results obtained with such an approach have focused only on the stabilization problem and cannot handle the design objectives such as attenuation of the disturbances effect, tracking control, structured uncertainties and so on (Tanaka, et al., 1998). On the other hand, only few results are available in the literature concerning the tracking control problem (Kung and Li, 1997; Ying., 1999; Tseng, et al., 2001; Tseng and Chen, 2001) and very few of them are concerned with the uncertain systems.

In (Kung and $\mathrm{Li}, 1997)$ a fuzzy tracking controller design for discrete time systems is proposed using the feedback linearization technique. In the same way (Ying. 1999) has established a simple necessary and sufficient condition for determining local stability of the fuzzy systems and then derived a fuzzy tracking controller. In (Tseng, et al., 2001), the authors deal with the tracking problem for nonlinear systems described by T-S model, with external disturbances and measurement noise. In their work, an $\mathrm{H} \infty$ performance related to the tracking error for bounded reference inputs is formulated and a fuzzy observerbased fuzzy controller is also developed. In (Tseng and Chen, 2001) the same strategy has been developed for a particular class of the nonlinear interconnected systems. 
Concerning uncertain systems, the tracking problem still remains open in the case of uncertain T-S fuzzy model. For this class of systems, we find some works dealing only with the stabilisation problem and stability analysis (Tanaka, et al., 1996; Lee, et al., 2001). This work is on the line of the one presented by (Tseng, et al., 2001), but our contribution concerns the case of uncertain T-S Fuzzy models with external disturbances.

Hence, this paper presents a novel approach based on the T-S fuzzy model representation to deal with the problem of tracking control for non linear dynamic system with bounded external disturbances and admissible uncertainties using T-S fuzzy model. In this approach, LMI-based design problems are defined and employed to find feedback gains of a fuzzy controller and common positive definite matrices $\mathrm{P}$ satisfying a stability criterion derived in terms of Lyapunov direct method. Based on this control scheme and that criterion, a fuzzy controller is then designed to stabilize an augmented system and to achieve the $\mathrm{H} \infty$ control performance.

This paper is organised as follows: Section 2 introduces the uncertain T-S fuzzy model and gives the proposed control law. In Section 3, a sufficient condition is developed to ensure the stability of the augmented system with a reference model. Following this, a method and some manipulations are proposed to improve the LMI feasibility of the main result presented in the second theorem. A simulation example is proposed in Section 4.

\section{PROBLEM FORMULATION}

The Takagi-Sugeno fuzzy dynamic model is described by fuzzy IF-THEN rules, which locally represent linear input-output relations of nonlinear systems. Consider the $\mathrm{i}$ th rule of uncertain and perturbed fuzzy model described as follows (Takagi and Sugeno, 1985):

\section{Plant Rule $\boldsymbol{i}$ :}

If $z_{1}(t)$ is $F_{i 1}$ and $\ldots$ and $z_{p}(t)$ is $F_{i p}$

Then $\dot{x}=\left(A_{i}+\Delta A_{i}\right) x(t)+\left(B_{i}+\Delta B_{i}\right) u(t)+\varphi(t)$

$$
i=1,2, \ldots, r \text {. }
$$

where $F_{i j}$ is the fuzzy set and $r$ is the number of IfThen rules. $x(t) \in R^{n}$ is the state vector, $u(t) \in \boldsymbol{R}^{m}$ is the input vector, $\varphi(t)$ is the unknown external disturbance with a known upper bound $\varphi_{\text {up }} \geq\|\varphi(t)\|$, $z_{1}(t) \sim z_{p}(t)$ are the premise variables that are functions of the states, $A_{i} \in \boldsymbol{R}^{n \times n}$, and $B_{i} \in \boldsymbol{R}^{n \times m}$ describe the nominal system and $\Delta A_{i}, \Delta B_{i}$ represent the time varying parameter uncertainties which are defined as follows:

$$
\left[\Delta A_{i}(t) \quad \Delta B_{i}(t)\right]=H \quad F(t)\left[\begin{array}{ll}
E_{1 i} & E_{2 i}
\end{array}\right] \quad i=1,2, \ldots, r .
$$

where $H, \quad E_{1 i}$ and $E_{2 i} i=1,2, \ldots, r$, are known constant real matrices with appropriate dimensions, and $F(t)$ is unknown matrix function which is bounded by :

$$
F(t) \in \Omega:=\left\{F(t) / F(t)^{T} F(t) \leq I\right\}
$$

Note that the element of $F(t)$ are Lebesgue measurable.

Given a pair of $(x(t), u(t))$, the final outputs of the fuzzy systems are inferred as follows :

$$
\begin{aligned}
\dot{x}(t) & =\frac{\sum_{i=1}^{r} w_{i}(z(t))\left\{\left(A_{i}+\Delta A_{i}\right) x(t)+\left(B_{i}+\Delta B_{i}\right) u(t)+\varphi(t)\right\}}{\sum_{i=1}^{r} w_{i}(z(t))} \\
& =\sum_{i=1}^{r} h_{i}(z(t))\left\{\left(A_{i}+\Delta A_{i}\right) x(t)+\left(B_{i}+\Delta B_{i}\right) u(t)\right\}+\varphi(t)
\end{aligned}
$$

where

$$
\begin{gathered}
z(t)=\left[\begin{array}{llll}
z_{1}(t) & z_{2}(t) & \ldots & z_{p}(t)
\end{array}\right], \\
w_{i}(z(t))=\prod_{j=1}^{p} F_{i j}\left(z_{j}(t)\right)
\end{gathered}
$$

and for all $t$,

$$
\begin{aligned}
& \left\{\begin{array}{l}
\sum_{i=1}^{r} w_{i}(z(t))>0 \\
w_{i}(z(t)) \geq 0
\end{array} \quad i=1,2, \ldots, r(5) .\right. \\
& h_{i}(z(t))=\frac{w_{i}(z(t))}{\sum_{i=1}^{r} w_{i}(z(t))}
\end{aligned}
$$

where, $F_{i j}\left(z_{j}(t)\right)$ is the degree of membership of $z_{j}(t)$ to $F_{i j}$, and for all $t$, we have (Kim, et al., 1995; Tanaka, et al., 1998; Wang, et al., 1995; Wang, et al., 1996)

$$
\left\{\begin{array}{l}
\sum_{i=1}^{r} h_{i}(z(t))=1 \\
h_{i}(z(t)) \geq 0
\end{array} \quad i=1,2, \ldots, r\right.
$$

To deal the tracking problem for such system, we consider the following reference model (Narendra and Annaswamy, 1989; Sastry and Bodson, 1989):

$$
\dot{x}_{r}=A_{r} X_{r}(t)+r(t),
$$

where $x_{r}(t)$ is the reference state, $A_{r}$ is a specific asymptotically stable matrix, and $r(t)$ is a bounded reference input.

Let us consider the $\mathrm{H} \infty$ tracking performance related to tracking error $x_{r}(t)-x(t)$ as follows (Tseng, et al., 2001; Tseng and Chen, 2001; Chen, 1996) (Essounbouli, et al., 2002):

$$
\frac{\int_{0}^{t f}\left\{\left[x_{r}(t)-x(t)\right]{ }^{T} Q\left[x_{r}(t)-x(t)\right]\right\} d t}{\int_{0}^{t f}\left\{r(t)^{T} r(t)+\varphi(t)^{T} \varphi(t)\right\} d t} \leq \eta^{2}
$$

or

$$
\begin{aligned}
\int_{0}^{t f}\left\{\left[x_{r}(t)-x(t)\right]^{T} Q\left[x_{r}(t)-x(t)\right]\right\} d t \\
\leq \eta^{2} \int_{0}^{t f}\left\{r(t)^{T} r(t)+\varphi(t)^{T} \varphi(t)\right\} d t
\end{aligned}
$$


Where $t_{f}$ is the terminal time of control, $Q$ a positive definite weighting matrix, and $\eta$ is a prescribed attenuation level.

If we choose the following control law for the $i$ th rule

\section{Control Rule $\boldsymbol{i}$ :}

If $z_{1}(t)$ is $F_{i 1}$ and $\ldots$ and $z_{p}(t)$ is $F_{i p}$

Then $u(t)=-K_{i}\left[x_{r}(t)-x(t)\right] \quad i=1,2, \ldots, r$

Then the overall fuzzy controller is given by

$$
\begin{aligned}
u(t) & =-\frac{\sum_{i=1}^{r} w_{i}(z(t)) K_{i}\left[x_{r}(t)-x(t)\right]}{\sum_{i=1}^{r} w_{i}(z(t))} \\
& =-\sum_{i=1}^{r} h_{i}(z(t)) K_{i}\left[x_{r}(t)-x(t)\right]
\end{aligned}
$$

By applying the control law above and considering the condition (3), the dynamics $x(t)$ and $x_{r}(t)$ (i.e. the state and the reference variable respectively) lead to the following augmented system:

$$
\dot{\widetilde{x}}=\left[A_{a}+H_{a} F_{a}(t) E_{a}\right] \tilde{x}(t)+\phi(t)
$$

where

$$
\begin{gathered}
A_{a}=\sum_{i=1}^{r} \sum_{j=1}^{r} h_{i} h_{j}\left[\begin{array}{cc}
A_{i}+B_{i} K_{j} & -B_{i} K_{j} \\
0 & A_{r}
\end{array}\right] \\
E_{a}=\sum_{i=1}^{r} \sum_{j=1}^{r} h_{i} h_{j}\left[\begin{array}{cc}
E_{1 i}+E_{2 i} K_{j} & -E_{2 i} K_{j} \\
0 & 0
\end{array}\right] \\
H_{a}=\left[\begin{array}{ll}
H & 0 \\
0 & 0
\end{array}\right] \quad F_{a}(t)=\left[\begin{array}{cc}
F(t) & 0 \\
0 & 0
\end{array}\right] \\
\phi(t)=\left[\begin{array}{l}
\varphi(t) \\
r(t)
\end{array}\right] \quad \text { and } \quad \tilde{x}=\left[\begin{array}{c}
x \\
x_{r}
\end{array}\right]
\end{gathered}
$$

Hence, the Ho tracking performance in (9) can be modified as follows:

$$
\begin{gathered}
\int_{0}^{t f}\left\{\left[x_{r}(t)-x(t)\right]^{T} Q\left[x_{r}(t)-x(t)\right]\right\} d t=\int_{0}^{t f} \widetilde{x}^{T}(\mathrm{t}) \widetilde{Q} \widetilde{x}(t) d t \\
\leq \eta^{2} \int_{0}^{t f} \phi(t)^{T} \phi(t) d t
\end{gathered}
$$

where $Q$ is a symmetric positive definite weighting matrix and

$$
\widetilde{Q}=\left[\begin{array}{cc}
Q & -Q \\
-Q & Q
\end{array}\right]
$$

and,

$$
\phi(t)^{T} \phi(t)=r(t)^{T} r(t)+\varphi(t)^{T} \varphi(t)
$$

\section{FUZZY TRACKING CONTROL DESIGN FOR AN UNCERTAIN SYSTEM :}

In this study, the objective is to determine the gains of the fuzzy control law (11) that achieve the $\mathrm{H} \infty$ tracking control performance in (14). Consequently we can state the following results.

\section{Theorem 1}

If there exist a symmetric positive definite matrix $P=$ $P^{T}>0$, positive constants $\tau$ and $\eta$, and the feedback gains $K_{i}$ shown in (11), such that the following condition (15) hold :

$\left[\begin{array}{ccc}A_{a}^{T} P+P A_{a}+\widetilde{Q}+\tau E_{a}^{T} E_{a} & P H_{a} & P \\ H_{a}^{T} P & -\tau I & 0 \\ P & 0 & -\eta^{2} I\end{array}\right] \leq 0$

then the closed loop fuzzy system (13) is quadratically stable and the $\mathrm{H} \infty$ tracking control performance in (14) is guaranteed for a prescribed attenuation $\eta$.

\section{Proof :}

The augmented system (13) can be modified as follow:

$$
\dot{\widetilde{x}}=A_{a} \widetilde{x}(t)+H_{a} q(t)+\phi(t)
$$

with :

Note that

$$
q(t)=F_{a}(t) E_{a} \tilde{x}(t)
$$

$$
F(t)^{T} F(t) \leq I \quad \Leftrightarrow \quad F_{a}(t)^{T} F_{a}(t) \leq I
$$

From (17) and (18), we can write

$$
q(t)^{T} q(t) \leq\left(E_{a} \widetilde{x}(t)\right)^{T}\left(E_{a} \widetilde{x}(t)\right)=\widetilde{x}(t)^{T} E_{a}^{T} E_{a} \widetilde{x}(t)
$$

Let us now consider the following candidate Lyapunov function for the augmented system (13) :

$$
V=\widetilde{x}^{T}(t) P \tilde{x}(t)
$$

with $P=P^{T}>0$.

then, the augmented system (13) is quadratically stable and the $\mathrm{H}_{\infty}$ tracking performance in (14) is guaranteed if

$$
\frac{d V(\tilde{x}, t)}{d t}+\widetilde{x} \widetilde{Q} \widetilde{x}-\eta^{2} \phi^{T} \phi \leq 0
$$

Using (16) and the derivative of (20), the condition above becomes.

$$
\begin{aligned}
\widetilde{x}^{T}\left(A_{a}^{T} P+P A_{a}+\tilde{Q}\right) \tilde{x}+ & q(t)^{T} H_{a}^{T} P \widetilde{x}+\widetilde{x}^{T} P H_{a} q(t) \\
& +\phi^{T} P \widetilde{x}+\widetilde{x}^{T} P \phi-\eta^{2} \phi^{T} \phi \leq 0
\end{aligned}
$$

From (17) and (20), the conditions (19) and (21) can be written as :

$$
\left[\begin{array}{c}
\widetilde{x}(t) \\
q(t) \\
\phi(t)
\end{array}\right]^{T}\left[\begin{array}{ccc}
-E_{a}^{T} E_{a} & 0 & 0 \\
0 & I & 0 \\
0 & 0 & 0
\end{array}\right]\left[\begin{array}{c}
\tilde{x}(t) \\
q(t) \\
\phi(t)
\end{array}\right] \leq 0
$$

$$
\left[\begin{array}{c}
\widetilde{x}(t) \\
q(t) \\
\phi(t)
\end{array}\right]^{T}\left[\begin{array}{ccc}
A_{a}^{T} P+P A_{a}+\widetilde{Q} & P H_{a} & P \\
H_{a}^{T} P & 0 & 0 \\
P & 0 & \eta^{2} I
\end{array}\right]\left[\begin{array}{c}
\tilde{x}(t) \\
q(t) \\
\phi(t)
\end{array}\right] \leq 0(24)
$$

By using the S-procedure (Boyd, et al., 1994) subject to the existence of a positive scalar $\tau>0$, the conditions (23) and (24) lead to: 


$$
\left[\begin{array}{ccc}
A_{a}^{T} P+P A_{a}+\widetilde{Q}+\tau E_{a}^{T} E_{a} & P H_{a} & P \\
H_{a}^{T} P & -\tau I & 0 \\
P & 0 & -\eta^{2} I
\end{array}\right] \leq 0
$$

This condition that ensures the quadratic stability of the closed loop fuzzy system, is not easily taken in to deduce the gains control. Thus, in view to find a more exploitable mathematical condition for the robust performance problem for the uncertain system, we choose:

$$
\begin{gathered}
A_{i j}=\left[\begin{array}{cc}
A_{i}+B_{i} K_{j} & -B_{i} K_{j} \\
0 & A_{r}
\end{array}\right] \\
E_{a i j}=\left[\begin{array}{cc}
E_{1 i}+E_{2 i} K_{j} & -E_{2 i} K_{j} \\
0 & 0
\end{array}\right]
\end{gathered}
$$

In this case, the following result can be announced

\section{Theorem 2}

If there exist a symmetric positive definite matrix $P=$ $P^{T}>0$, positive constants $\tau$ and $\eta$, and the feedback gains $K_{i}$ shown in (11), such that the following condition (27) hold:

$$
\left[\begin{array}{cccc}
A_{i j}^{T} P+P A_{i j}+\widetilde{Q} & P H_{a} & P & E_{a i j}^{T} \\
H_{a}^{T} P & -\tau I & 0 & 0 \\
P & 0 & -\eta^{2} I & 0 \\
E_{a i j} & 0 & 0 & -\tau^{-1} I
\end{array}\right] \leq 0
$$$$
i, j=1,2, . ., r \quad(27)
$$

then the closed loop fuzzy system (13) is quadratically stable and the $\mathrm{H} \infty$ tracking control performance in (14) is guaranteed for a prescribed attenuation $\eta$.

\section{Proof}

By using the terms (25) and (26), the condition (15) in theorem 1 will be written as

$$
\sum_{i=1}^{r} \sum_{j=1}^{r} h_{i}(z(t)) h_{j}(z(t))\left[\begin{array}{ccc}
A_{i j}^{T} P+P A_{i j}+\tilde{Q} & P H_{a} & P \\
+\tau E_{a i j}^{T} E_{a i j} & & \\
H_{a}^{T} P & -\tau I & 0 \\
P & 0 & -\eta^{2} I
\end{array}\right] \leq 0
$$

This is equivalent to

$$
\left[\begin{array}{ccc}
A_{i j}^{T} P+P A_{i j}+\widetilde{Q}+\tau E_{i j}^{T} E_{i j} & P H_{a} & P \\
H_{a}^{T} P & -\tau I & 0 \\
P & 0 & -\eta^{2} I
\end{array}\right] \leq 0
$$

With some manipulation and using the Schur complement (Boyd, et al., 1994) the condition above is equivalent to that in theorem 2 (27).

Solving the conditions of theorem 2 remains a difficult task. Therefore, in this section we will first show how to fulfil this last inequality in terms of LMIs (standard LMI). Note that, for the convenience of design, we assume that:
$P=\left[\begin{array}{cc}P_{1} & 0 \\ 0 & P_{2}\end{array}\right]$ with $P_{1}=P_{1}^{T}>0$. and $P_{2}=P_{2}{ }^{T}>0$

The condition (27) of theorem 2 becomes

$$
\left[\begin{array}{llllllll}
H_{11} & H_{12} & H_{13} & 0 & H_{15} & 0 & H_{17} & 0 \\
H_{21} & A_{r}^{T} P_{2} & 0 & 0 & 0 & P_{2} & H_{27} & 0 \\
H_{31} & 0 & -\tau I & 0 & 0 & 0 & 0 & 0 \\
0 & 0 & 0 & -\tau I & 0 & 0 & 0 & 0 \\
H_{51} & 0 & 0 & 0 & -\eta^{2} I & 0 & 0 & 0 \\
0 & P_{2} & 0 & 0 & 0 & -\eta^{2} I & 0 & 0 \\
H_{71} & H_{72} & 0 & 0 & 0 & 0 & -\tau^{-1} I & 0 \\
0 & 0 & 0 & 0 & 0 & 0 & 0 & -\tau^{-1} I
\end{array}\right] \leq 0
$$

with

$$
\begin{aligned}
& H_{11}=\left(A_{i}+B_{i} K_{j}\right)^{T} P_{1}+P_{1}\left(A_{i}+B_{i} K_{j}\right)+Q \\
& H_{12}=H_{21}^{T}=-P_{1} B_{i} K_{j}-Q \\
& H_{13}=H_{31}^{T}=P_{1} H \\
& H_{15}=H_{51}=P_{1} \\
& H_{17}=H_{71}^{T}=\left(E_{1 i}+E_{2 i} K_{j}\right)^{T} \\
& H_{27}=H_{72}^{T}=\left(E_{2 i} K_{j}\right)^{T}
\end{aligned}
$$

$P_{1}, P_{2}$ and $K_{i}$ can be solved following these two steps First, note that (30) implies that

$$
\begin{array}{r}
H_{11}=\left(A_{i}+B_{i} K_{j}\right)^{T} P_{1}+P_{1}\left(A_{i}+B_{i} K_{j}\right)+Q \leq 0 \\
i, j=1,2, \ldots, r
\end{array}
$$

After pre-post-multiply (31) by $N_{1}=P_{1}^{-1}$ and letting $Y_{i}=K_{i} N_{1}$, we obtain

$N_{1} A_{i}^{T}+Y_{j}^{T} B_{i}^{T}+A_{i} N_{1}+B_{i} Y_{j}+N_{1} Q N_{1} \leq 0 \quad i, j=1,2, . ., r$ By using the Schur complement, we get

$$
\begin{gathered}
{\left[\begin{array}{cc}
N_{1} A_{i}^{T}+Y_{j}^{T} B_{i}^{T}+A_{i} N_{1}+B_{i} Y_{j} & N_{1} \\
N_{1} & -Q^{-1}
\end{array}\right] \leq 0} \\
i, j=1,2, \ldots, r
\end{gathered}
$$

The variables $N_{1}$ and $Y_{i}$ (thus $P_{1}=N_{1}^{-1}$ and $\left.K_{i}=Y_{i} N_{1}^{-1}\right)$ can be obtained by solving the LMI (32).

In the second step, by substituting $P_{1}$ and $K_{i}$ into (30) which will be a standard linear matrix inequality (LMI's) (Gahinet, et al., 1995), we can easily solve $P_{2}$ and $\tau$ from (30). If there exist positive definite solutions $P_{1}, P_{2}$ and a positive scalar $\tau$ such that (30) holds, then the closed loop system (12) is stable and the $\mathrm{H} \infty$ tracking performance in (14) can be achieved for a prescribed attenuation level $\eta$.

\section{EXAMPLE}

Let us consider the problem of the tracking on an inverted pendulum with a cart. The equations of motion for the pendulum are (Cannon, 1967) 
$\dot{x}_{1}=x_{2}$

$\dot{x}_{2}=\frac{g \sin \left(x_{1}\right)-a m l x_{2}^{2} \sin \left(2 x_{1}\right) / 2-a \cos \left(x_{1}\right) u}{4 l / 3-a m l \cos ^{2}\left(x_{1}\right)}$

where $x_{1}$ denotes the angle (in radians) of the pendulum from the vertical, $x_{2}$ is the angular velocity, $g=9.8 \mathrm{~m} / \mathrm{s}^{2}$ is the constant gravity, $m$ is the mass of the pendulum, $M$ is the mass of the cart, $2 l$ is the length of the pendulum, and $u$ is the force applied to the cart (in Newtons). $a=1 /(m+M)$. Let $m$ $=0.1 \mathrm{Kg}, M=1 \mathrm{Kg}, 2 \mathrm{l}=1.0 \mathrm{~m}$.

To minimize the design effort and complexity, we try to use as few rules as possible. Notice that when $x_{1}= \pm \pi / 2$, the system is uncontrollable. Hence, we approximate the system by the following two-rule T$\mathrm{S}$ fuzzy model, where the membership functions are shown in Figure 1.

Rule 1 : If $x_{1}(t)$ is about 0

Then

$\dot{x}(t)=\left(A_{1}+\Delta A_{1}\right) x(t)+\left(B_{1}+\Delta B_{1}\right) u(t)+\varphi(t)$

Rule 2 : If $x_{1}(t)$ is about $x_{1}= \pm \pi / 2\left(\left|x_{1}(t)\right|<2\right)$

\section{Then}

$\dot{x}(t)=\left(A_{2}+\Delta A_{2}\right) x(t)+\left(B_{2}+\Delta B_{2}\right) u(t)+\varphi(t)$

where

$$
\begin{aligned}
& A_{1}=\left[\begin{array}{cc}
0 & 1 \\
g / 4 l / 3-a m l & 0
\end{array}\right] \\
& B_{1}=\left[\begin{array}{c}
0 \\
-a / 4 l / 3-a m l
\end{array}\right] \\
& A_{2}=\left[\begin{array}{cc}
0 \\
2 g / \pi\left(4 l / 3-a m l \beta^{2}\right) & 0
\end{array}\right] \\
& B_{2}=\left[\begin{array}{c}
0 \\
-a \beta / 4 l / 3-a m l \beta^{2}
\end{array}\right]
\end{aligned}
$$

with $\beta=\cos \left(88^{\circ}\right)$ and

$$
\begin{aligned}
& \Delta A_{1}=H F(t) E_{11} \quad \Delta A_{2}=H F(t) E_{12} \\
& \Delta B_{1}=H F(t) E_{21} \quad \Delta B_{2}=H F(t) E_{22} \\
& H=\left[\begin{array}{l}
0 \\
1
\end{array}\right], \quad F(t)=\sin (2 t) \in \Omega \\
& E_{11}=\left[\begin{array}{ll}
1 & 0
\end{array}\right], \\
& E_{12}=\left[\begin{array}{ll}
1 & 0
\end{array}\right], \\
& \varphi(t)=\left[\begin{array}{lll}
0.01 \sin (2 t) & E_{21}=0.5 \quad(i=1)
\end{array}\right. \\
& 0.02 \cos (2 t)]^{T} .
\end{aligned}
$$

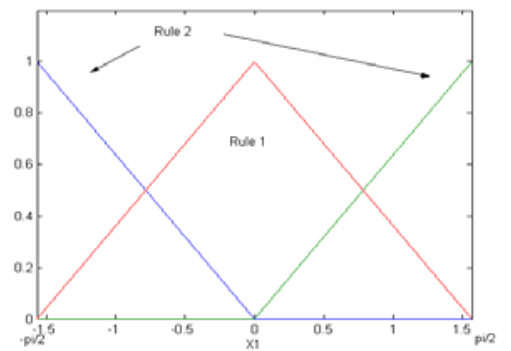

Fig. 1. Membership functions of two-rule model
Afterwards, we compute the gains $K_{i}$ according to the steps defined in section 3 for the conditions (27). Hence, by choosing

$$
\begin{aligned}
Q=\left[\begin{array}{cc}
1 e^{-8} & 0 \\
0 & 9 e^{-7}
\end{array}\right], A_{r} & =\left[\begin{array}{cc}
0 & 1 \\
-6 & -5
\end{array}\right] \\
\text { and } \quad r(t) & =\left[\begin{array}{c}
0 \\
0.7381 \sin (t)
\end{array}\right],
\end{aligned}
$$

The following gains are obtained

$K_{1}=\left[\begin{array}{ll}416.0733 & 300\end{array}\right], \quad K_{2}=\left[\begin{array}{ll}416.0733 & 300\end{array}\right]$ and the attenuation level is $\eta=0.5$.

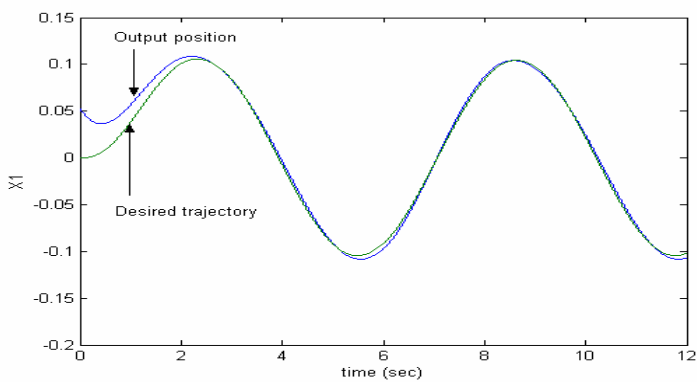

Fig. 2. Position tracking

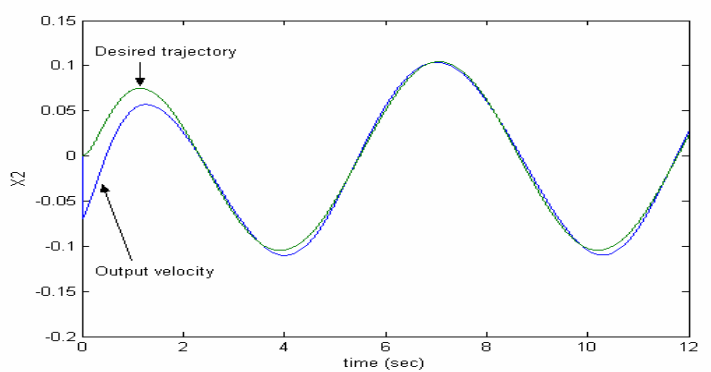

Fig. 3. Velocity Tracking

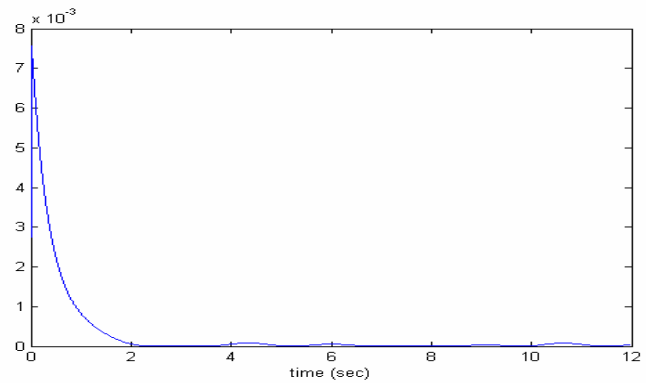

Fig. 4. Quadratic error tracking

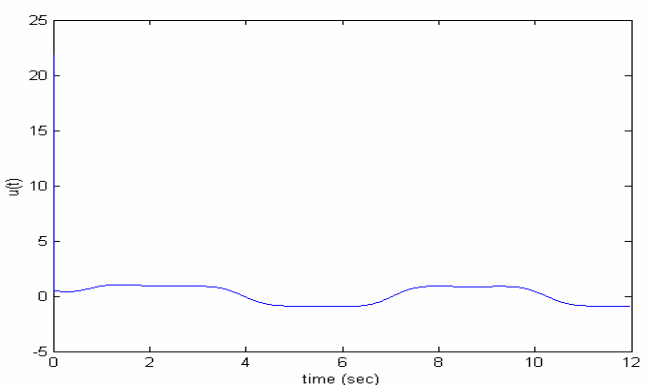

Fig. 5. Control force

Figure 2 shows the position tracking trajectory with as initial conditions $x_{1}(0)=0.05$ and $x_{2}(0)=0$ for the system, Figure 3 illustrates the tracking velocity signal. Figure 4 shows the quadratic error signal whereas the force control signal is given in figure 5 . 
These obtained results show a good tracking performance for the case of an uncertain and a perturbed system.

\section{CONCLUSION}

In this paper, we have developed via $\mathrm{H} \infty$ a controller design method for nonlinear uncertain systems with external disturbances described by Takagi-Sugeno model. Using a tracking reference model, a stability condition scheme based on an augmented system with a guaranteed $\mathrm{H} \infty$ performance was proposed to ensure good tracking performances. The main results are concerning the two developed theorems to ensure a sufficient condition in terms of LMI for the problem of uncertain systems tracking, expressed in Takagi Sugeno form.

\section{REFERENCES}

Boyd S., L. El Ghaoui, E. Feron, and V. Balakrishnan (1994) Linear Matrix Inequalities in System and Control Theory. PA: SIAM Philadelphia, 1994.

Cannon R. H. (1967). Dynamics of Physical Systems. McGraw-Hill, New York,1967.

Chen, B. S., C. H Lee, and. Y. C Chang. (1996). Hळ tracking design of uncertain non-linear SISO systems: Adaptive fuzzy approach. IEEE Trans. Fuzzy Syst., vol. 4, no. 1, pp. 32-43.

Essounbouli, N., A. Hamzaoui and J. Zaytoon (2002) A supervisory robust adaptive fuzzy controller in Proc. of $15^{\text {th }}$ IFAC World Congress on Automatic and Control, Barcelona, Spain.

Gahinet, P, A. Nemirovski, A. J. Laub, and M. Chilali (1995). LMI Control Toolbox". Natick, MA: MathWorks.

Hopp, T. H., and W. E. Schmitendore, (1990). Design of a Linear Controller for Robust Tracking and Model Following. ASME Journal of Dynamic Systems, Measurement, and Control, Vol 112, pp 552-558.

Kim: W. C, S. C. Ahn, W. H. Kwon (1995). Stability Analysis and Stabilisation of Fuzzy state space Models. Fuzzy Set and Systems. no. 71, pp. 131142.

Kung, C.C, and H. H. Li (1997). Tracking control of non linear systems by fuzzy model-based controller. in Proc. IEEE Int. Conf., vol. 2, July 1997, pp. 623-628.

Lee, K. R, E. T. Jeung, H. B. Park (2001). Robust Fuzzy $\mathrm{H} \infty$ Control for Uncertain Nonlinear Systems via State Feedback: an LMI approach Elsevier Fuzzy Sets and Systems 120 123-134

Narendra, K. S and A. M. Annaswamy (1989). Stable Adaptive Systems. Englewood Cliffs, NJ: Prentice-Hall.

Sastry, S and M. Bodson (1989). Adaptive Control Stability, Convergence and Robustness. Englewood Cliffs, NJ: Prentice-Hall.

Takagi T and M. Sugeno (1985). Fuzzy identification of systems and its application to modelling and control. IEEE Trans. Syst., Man and Cyber., vol.1115, pp. 116-132.
Tanaka, K., T. Ikeda and H. O. Wang (1998) Fuzzy regulators and fuzzy observers: Relaxed stability conditions and LMI-based designs. IEEE Trans., Fuzzy, Sys., vol. 6 n², May.

Tanaka, K., T. Ikeda, and Hua O. Wang (1996). Robust Stabilzation of a Class of Uncertain Nonlinear Systems via Fuzzy Control: Quadratic Stabilizability, Hळ Control Theory, and Linear Matrix Inequalities. IEEE Trans., Fuzzy, Sys., vol. $4 \mathrm{n}^{\circ} 1$.

Tseng, C. and B. Chen (2001). Hळ decentralized fuzzy model reference tracking control design for non linear interconnected systems. IEEE Trans., Fuzzy, Sys., vol. 9 n ${ }^{\circ} 6$.

Tseng, C.,B. Chen and H. J. Uang (2001)Fuzzy tracking Control Design for nonlinear Dynamic systems via t-S fuzzy model. IEEE Trans., Fuzzy, Sys., vol. $9 \mathrm{n}^{\circ} 3$.

Wang, H. O., K. Tanaka and M. Griffin (1995). Parallel Distributed Compensation of Nonlinear Systems by Takagi-Sugeno Fuzzy Model. Proc. Fuzz IEEE/IFES '95, pp. 531-538.

Wang, H.O., K. Tanaka, and M. Griffin (1996). An approach to fuzzy control of non linear systems: Stability and design issues. IEEE Transactions on Fuzzy Systems, volume 4, pp 14-23.

Wu, H. S. and G. Leitman, (2000). Robust tracking and model following control with zero tracking error for uncertain dynamical systems. Journal of optimization theory an applications: Vol 107, No 1, pp. 169-182.

Ying,, H. (1999). Analytical analysis and feedback linearization tracking control of the general Takagi-Sugeno fuzzy dynamic systems. IEEE Trans. Syst., Man, Cybern., vol. 29, pp 290-298. 